\title{
Digital approaches in electronic biochips
}

\author{
C. Guiducci, E. Accastelli, F. M. Spiga \\ Laboratory of Life Sciences Electronics (CLSE), \\ Ecole Polytechnique Fédérale de Lausanne (EPFL), Lausanne, Switzerland \\ Tel: +41 (0)21 69 37813, Fax: +41 (0)2169 31105, Email: carlotta.guiducci@epfl.ch
}

\begin{abstract}
Electronic biochips are defined by the merge of integrated electronics, containing diverse sensors, with reaction solution and sample hold in reaction chamber(s). Based on their overall structure, electronic biochips can work in either well-confined or in area-confined configurations. Extreme parceling of the sample (digital approach), coupled with electronic biochips, can radically enhance the throughput performance of the assay, especially in an area-defined configuration. This takes advantage of the increased sensitivity that follows device miniaturization, as illustrated in our experiments on Silicon Nano Ribbons.
\end{abstract}

\section{Introduction}

Electronic biochips are characterized by the integration on one silicon substrate of a 2D collection of micro/nanosensors exposed to the wet environment (reaction chamber) and of the corresponding addressing, reading and processing circuitry. With respect to the existing devices addressed to bioanalytics for limited-volume samples - mainly based on fluorophore techniques or on passive chips featuring few electrodes - electronic biochips lead to significant improvements. First, by avoiding the use of optical scanners they enable fully-electronic implementation of analytical systems, therefore allowing high degree of autonomy, limited costs and high compactness. Besides point-of-care settings, several applications requiring high-throughput can be radically revolutionized. In fact, integrated sensing arrays enable fast readout and are in principle suitable for ultra-large scale implementations. Recent remarkable examples of ICsbased implementations are a self-contained point-of-care system for quantitative PCR (1) and the high-throughput DNA sequencing on-chip (2), which have been presented in the last two years.

The abovementioned systems, based on ISFETs (ion sensitive field effect transistors), work in a well-confined configuration (Figure 1, left) which is also common in existing fluorescence-based systems (3). In this approach a volume of solution, in which a specific reaction is designed to occur only in the presence of the molecule of interest, is delivered to a well sitting on a solid-state sensor. In the ISFET-based quantitative PCR system (1) the amount of synthesized DNA is measured through the increase of protons $\left(\mathrm{H}^{+}\right.$ions $)$in the solutions (i.e. decrease of $\mathrm{pH}$ ). This is taking advantage of the side-production of one proton per bound nucleotide in the polymerization reaction driven by the DNA polymerase on the template DNA. The miniaturized ISFET placed at the bottom of the well thus measures the $\mathrm{pH}$ variation, allowing label-free and real time quantification of the DNA polymerization, which in turn provides a quantification of the initial DNA present in the well. Whether by means of automatized or manual delivery, the system can be parallelized to target many molecules at a time by simply employing multiple wells and sensors.

The different area-confined configuration (Figure 1, bottom) has the advantage of avoiding reaction volume specification and delivery, and characterizes DNA and protein microarrays (e.g.: Illumina, Affymetrix). In these setups, typically, capture molecules are immobilized onto specific microregions of the chip surface which is exposed to one single macro-chamber or to a fluidic channel. The analyte, present in the sample volume at a given concentration, is captured selectively on one specific region of the chip by the corresponding capture molecule. The molecular binding events take place directly on the sensor surface modifying its interface properties (charge or impedance for field-effect sensors) and are transduced into an electrical signal.

Regardless of the choice of the measurement technique, both the above-mentioned configurations suffer intrinsically from specificity issues, since other molecular species are often present in the sample more abundantly than the molecules of interest (4). So far, fluorescence-based molecular techniques have been designed in support of bioassays with the purpose of reducing the interference and the artifacts generated from non-specific molecules in solution (3). For instance, the optical quantitative PCR currently use fluorescence/quencer labeled probes (e.g. TaqMan) that hybridize on the template, and that upon DNA polymerization are released and thus detected (5). Nevertheless, fluorescence is well known to have other drawbacks arising from poor stability of the dyes and their interference with the reactions, therefore it is far from representing a long-term solution. In the field of optical quantitative PCR, an revolutionary approach - digital PCR has been recently introduced for the well-confined 

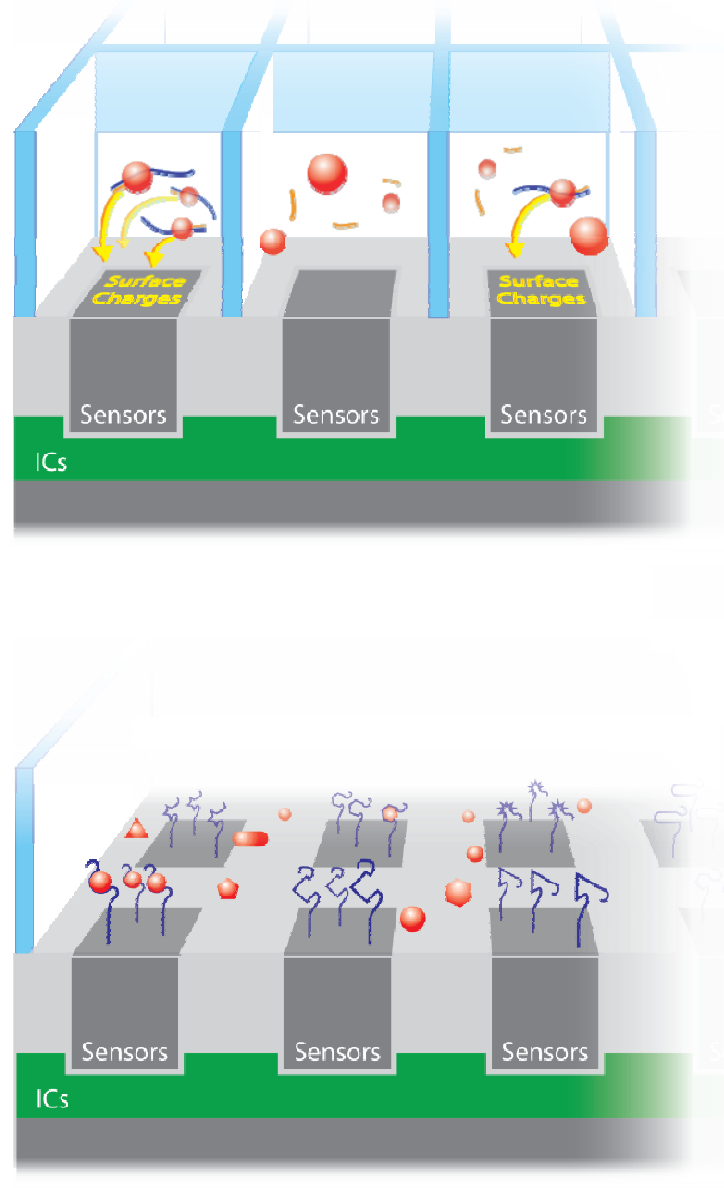

Figure 1 Top: Representation of an integrated electronic biochip working in a well-confined configuration. A chemical signal (yellow arrow) that causes a change in the surface charge is generated in the well (e.g.: by an enzyme, red sphere) only in the presence of a specific target molecule (purple strand).

Bottom: Representation of an electronic biochip working in the area-confined configuration. Each region of the chip active-surface is functionalized with specific molecular probes (purple string) designed to capture analyte molecules in solution (red shapes; each shape fits into only one purple strand type, according to their shape). The surface density of the captured analytes at equilibrium depends on their concentration in the well.

configuration, with the aim of improving the specificity. It consists of separating the sample in ultra-small parcels (volume range of $9 \mathrm{pL}-33 \mathrm{~nL}$ ) (3). The effect of this extreme volume reduction is that statistically there are few wells with only one DNA template copy, and virtually none (or few) with more. In this way the effect of the presence of one single molecule of interest in a specific well is clearly distinguishable from the other volumes where only nonspecific molecules are present (see Figure 2, top). The digital approach, presently coupled with optical detection devices, requires very high number of features to guarantee statistical validity. At present-the throughput of digital PCR (i.e. on a big number of different targets) relies on the use of different fluorescent/quenched probes tailored on the analyzed targets, and is therefore limited. Although, this possibility has not been explored yet, the improved parallelism and fast addressing enabled by electronic biochips would enable multi-target digital assays with high throughput (6).
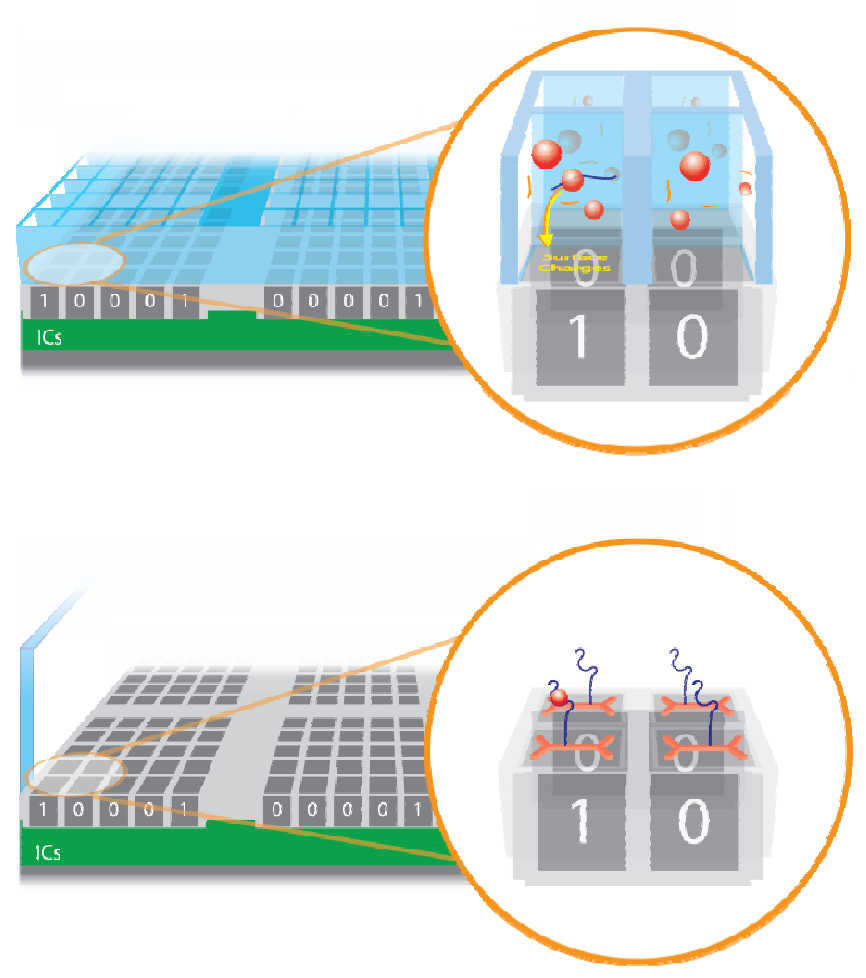

Figure 2 Top: Digital approach applied to an integrated biochip working in a well-confined configuration. The sample is divided in nanoliters-parcels in which one or no specific molecule is present. The reactions taking place in the well in presence of the analyte molecule (purple strand) generate a chemical signal over the background (e.g.: by an enzyme, red sphere) that is associated with the digital bit " 1 ". In absence of target molecules, no signal is generated, thus a " 0 " digital bit is consequently associated with the well. A suitable processing of the bit distribution results in the quantification of the concentration of the target molecule in the original sample.

Bottom: A digital implementation of a biochip working in an areaconfined configuration. Regions functionalized by one type of capture molecule (purple strand) specific for one target (red sphere) would feature several ultra-miniaturized sensors (in red; e.g.: silicon nanowires or nanoribbons). The higher sensitivity of nanosensors with respect to devices with larger footprint would allow a digital approach.

In our view, the digital approach coupled with fullyelectronic systems offers an even higher potential in areaconfined configurations (Figure 2, bottom). In fact, micro- 
regions functionalized with one type of capturing molecules could feature several smaller sensors which would detect one or no target molecule. Molecular dissociation time can thus be used as a distinctive feature since it is significantly longer for specific molecules with respect to non-specific ones (7, 8). In order to exploit this feature the system needs to be realtime capable -an intrinsic feature of field-effect-based sensing- as the dissociation times might differ for seconds.

Whether field effect devices could detect single molecules is still the object of an open debate, although remarkable detection performance has been presented in the recent years $(8,9)$. Even though issues related to noise remain crucial for the detection of a very low number of molecules per sensor, field effect devices present the interesting feature that size reduction does not imply a decreased sensitivity with respect to surface charge. Furthermore, sensitivity (current change vs. surface charge variation) is improved for devices with nanometric features and high surface to volume ratio (10, 11).

\section{Results}

In order to obtain data confirmation of the abovementioned sensitivity increase, we performed $\mathrm{pH}$ measurements on topdown $50 \mathrm{~nm}$ thick silicon nanoribbons (SiNRs) with different width. The SiNRs are fabricated in LETI-CEA (Grenoble) from Silicon-on-Insulator (SOI) wafers processed to obtain a $50 \mathrm{~nm}$ silicon top layer, and doped with Boron/Phosphorous to have n-type devices. The SiNRs are patterned using deep ultraviolet (DUV) or e beam lithography and etched by reactive ion etching (RIE). The SiNRs body is covered by a to high quality $3 \mathrm{~nm} \mathrm{SiO}$ gate oxide is grown by thermal dry oxidation while the rest of the chip is passivated by a multilayered insulator. The nanometric thickness of the silicon film (ideally smaller than the Thomas-Fermi screening length (12)), causes the electrical properties of the SiNRs to be highly impacted by the local environment, which can effectively induce a field effect that changes the carrier concentration, thus providing electrical transduction of the chemical interaction of interest occurring on the surface of the gate oxide. The SiNR chip has been coupled with a microfluidic setup that allows controlled real-time measurement in wet environment, as well as reduced solvents and reagents consumption and ease of integration with the SiNRs chip. The microchannels are realized with a chemical resistant double-coated tape, and are $190 \mu \mathrm{m}$ high. A Poly(methyl methacrylate) (PMMA) cap is placed on top to seal the channels and $\mathrm{Ag} / \mathrm{AgCl}$ inlets and outlets tubes inserted. These tubes are inserted into a microfluidic chip, serving both as REs and as inlet/outlet of the system (13).

The SiNRs were polarized by applying a gate voltage $\mathrm{V}_{\mathrm{FG}-\mathrm{S}}$ between the electrolyte solution, polarized by the REs, and the source diffusion. Figure 3 demonstrates the ability of the
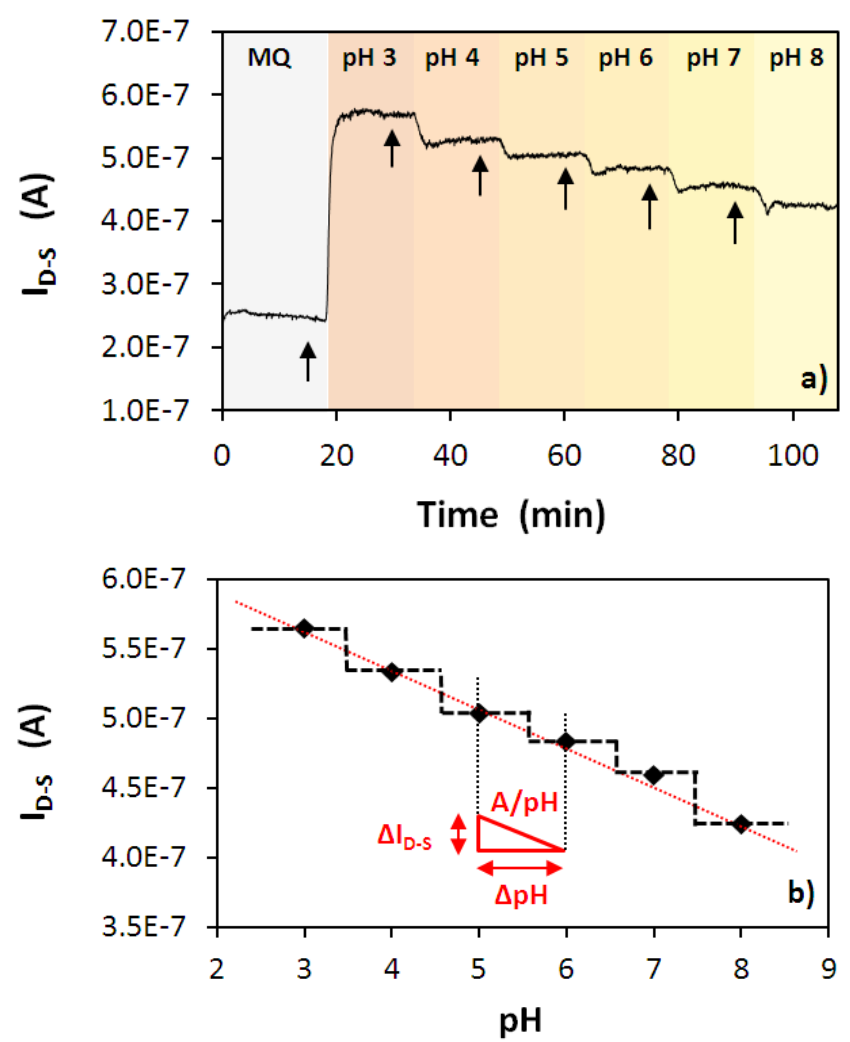

Figure 3 Top: Drain current $\left(\mathrm{I}_{\mathrm{D}-\mathrm{S}}\right)$ measurement in time upon injection of solutions with different $\mathrm{pH}$, and constant ionic strength. The arrows indicate the starting of the injections while the different regions on the plot indicate the time frame during which the SiNR is in contact with the previously injected solution (delay time due to tubings). The different $\mathrm{pH}$ solutions were prepared with $10 \mathrm{mM}$ phosphate buffers (PBS) containing $100 \mathrm{mM} \mathrm{KCl}$, which was added to stabilize the $\mathrm{Ag} / \mathrm{AgCl} \mathrm{RE}$. The first injection of ultrapure deionized water (MQ) defines a baseline and shows the influence of ionic strength on the measurement $\left(\mathrm{pH}_{\mathrm{MQ}} \sim 6.8\right)$. The flow rate was kept fixed at $5 \mu \mathrm{l} / \mathrm{min}$ during the whole experiment.

Bottom: the relationship between current change and $\mathrm{pH}$ change for a given device is obtained from (a) by averaging the value of ID-S over the injection time for each specific $\mathrm{pH}$ value and calculating the corresponding linear interpolation $\left(\mathrm{R}^{2}=0.99\right)$.

SiNRs to sense the concentration of protons $(\mathrm{pH})$. The change in the drain current upon injection of buffer solutions with different $\mathrm{pH}$ values is monitored in real-time. The change in surface charge density with the $\mathrm{pH}$ can be described by the site-binding model (14). In an n-type SiNR, increasing the $\mathrm{pH}$ leads to depletion of electrons carriers in the SiNR channel, leading to a decrease of its conductivity. $\mathrm{pH}$ measurements obtained with SiNRs of different width show that the reduction of the device width below $100 \mathrm{~nm}$ leads to an increase of the sensitivity (Figure 4). This data confirms that reducing the size of the device leads to an increased sensitivity with respect to surface charge. 


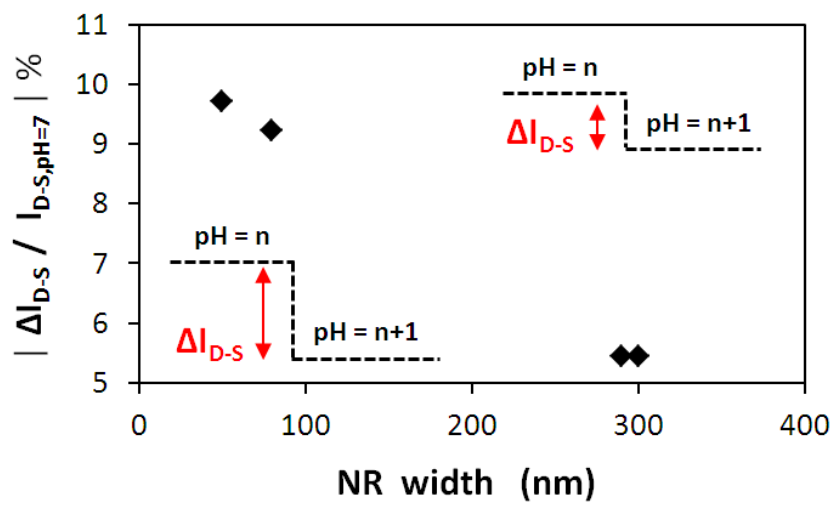

Figure 4 Normalized sensitivity of SiNR device vs. SiNR width. The sensitivity is expressed as the percent change of the drain current $\left(\Delta \mathrm{I}_{\mathrm{D}-\mathrm{S}}\right)$ with respect to $\mathrm{pH}$, normalized by the current at $\mathrm{pH}=7\left(\mathrm{I}_{\mathrm{D}-\mathrm{S}, \mathrm{pH}=7}\right)$.

\section{References}

(1) C. Toumazou, et al., "Simultaneous DNA amplification and detection using a $\mathrm{pH}$-sensing semiconductor system", 2013, Nat. Methods, vol. 10, pp. 641-646.

(2) J. M. Rothberg, et al., "An integrated semiconductor device enabling non-optical genome sequencing", Nature, 2011, vol. 475, pp. 348-352.

(3) E. Day, P.H. Dear, F. McCaughan, "Digital PCR strategies in the development and analysis of molecular biomarkers for personalized medicine", Methods 2013 59(1), pp. 101-107.

(4) D. Zichi, B. Eaton, B. Singer, L. Gold, "Proteomics and diagnostics: Let's Get Specific, again", Current Opinion in Chemical Biology, 2008, vol. 12-1, pp. 78-85

(5) R. H. Sedlak, K. R. Jerome, "Viral diagnostics in the era of digital polymerase chain reaction", Diagnostic Microbiology and Infectious Disease, 2013, Vol. 75- 1, pp 1-4.
(6) C. Guiducci and F.M. Spiga "Another transistor-based revolution: on-chip qPCR", Nature Methods, 2013, 10(7), pp. 617-8. (7) J. Gooding, "Nanoscale Biosensors: Significant Advantages over Larger Devices?", Small, 2006, vol. 2-3, pp. 313-315.

(8) A.K. Wanekaya, W. Chen, A. Mulchandani, N. V. Myung, "Nanowire-Based Electrochemical Biosensors," Electroanalysis, 2006, vol. 18, pp. 533-550.

(9) S. Sorgenfrei, C. Chiu, R. L. Gonzalez, Jr, Y. Yu, P. Kim, C. Nuckolls and K. L. Shepard, "Label-free single-molecule detection of DNA hybridization kinetics with a carbon nanotube field-effect transistor", Nature Nanotechnology, 2011, vol. 6, pp. 126-132.

(10) P. R. Nair and M. A. Alam, "Design Considerations of Silicon Nanowire Biosensors", IEEE Transactions on Electron Devices, 2007, vol. 54, pp. 3400-3408.

(11) N Elfström, AE Karlström, J Linnros," Silicon nanoribbons for electrical detection of biomolecules", Nano letters, 2008, vol. 8-3, pp. 945-949.

(12) O. Knopfmacher, A. Tarasov, W. Fu, M. Wipf, B. Niesen, M. Calame and C. Schönenberger, "Nernst Limit in Dual-Gated SiNanowire FET Sensors", Nano Letters, 2010, vol. 10, pp. 22682274 .

(13) E. Accastelli, G. Cappi, J. Buckley and C. Guiducci, "Comparison between front-and back-gating of Silicon Nanoribbons in real-time sensing experiments", The 13th IEEE International Conference on Nanotechnology, August 5-8, 2013, Beijing, China.

(14) D. E. Yates, S. Levine, T. W. Healy, "Site-binding Model of the Electrical Double Layer at the Oxide/Water Interface", Journ. Chem. Soc. of the Chemical Society, Faraday Transactions 1: Physical Chemistry in Condensed Phases, 1974, vol. 70, pp. 18071818.

\footnotetext{
${ }^{1}$ Quantitative PCR: standard analytical technique for the quantification of specific DNA molecules in samples (eg: virus quantification, genotyping).
} 